PoS $\quad \begin{aligned} & \text { PROCEEDINGS } \\ & \text { OF SCIENCE }\end{aligned}$

\title{
Preface to PSTP 2017 Proceedings
}

\section{Seongtae Park}

Chair of the Local Organizing Committee

Institute for Basic Science

E-mail: stpark@ibs.re.kr

XVII International Workshop on Polarized Sources, Targets \& Polarimetry

16-20 October 2017

Kaist, South Korea 
The 17th International Workshop on Polarized Sources, Targets and Polarimetry (PSTP 2017) was held at Lecture Hall at the Munji campus of the Korea Advanced Institute of Science and Technology (KAIST), Daejeon, South Korea from October 16 to 20, in 2017. It was sponsored jointly by the Institute for Basic Science (IBS) and the KAIST.

The Workshop continued a long tradition of bringing together experts in the technical fields necessary to carry out particle physics experiments involving spin at accelerators around the world. The three previous workshops in this series were held at Bochum, Germany in 2015, Charlottesville, USA in 2013 and St. Petersburg, Russia in 2011, following the tradition of circulating the Workshop among sites on different continents.

Total thirty seven scientists were registered and the topics covered at the workshop were:

- Polarized Solid Target

- Polarized Gas Target

- Polarized Electron Sources

- Polarized Ion Sources

- Proton Polarimetry

- Electron Polarimetry

- Applications of Spin

- New Initiatives

The Organizing Committee thank all the speakers and attendees for making this a memorable Workshop. We appreciate the financial support of the Institute for Basic Science (IBS), and KAIST for the support with venue place and other facilities. We also express our gratitude to the administrative staffs of the Center for Axion and Precision Physics Research of IBS (CAPP) for their excellent administrative support. 